\title{
Mus spretus Lataste: a hygienic house mouse?
}

\author{
JANE L. HURST \& JEREMY SMITH \\ Behaviour \& Ecology Research Group, Department of Life Science, University of Nottingham. \\ University Park, Nottingham NG7 2RD, U.K.
}

(Received 8 November 1993; initial acceptance 13 January 1994;

final acceptance 3 June 1994; MS. number: 4521)

\begin{abstract}
Mus spretus, an aboriginal species of house mouse, appears to show a pattern of behaviour not reported in other rodents. Faeces are picked up and carried short distances in the mouth or rolled along the ground with the tip of the snout, often repeatedly. Two experiments using wild-caught subjects held temporarily in captivity investigated the potential function of this behaviour. Provided with a choice of faeces up to $12 \mathrm{~h}$ old from different donors in an arena, mice showed a strong preference for manipulating their own fresh faeces deposited during the trial, especially those in corners. A second experiment, using single fresh faecal samples encountered in a tunnel, confirmed that mice manipulated their own faeces (in 56\% of trials) more frequently than those from conspecifics ( $26 \%$ of trials). Manipulation was not affected by the presence of tapeworm eggs in faeces, although this stimulated increased investigation from the donor. In both experiments, differences in investigation suggested that mice could discriminate between the faeces of conspecifics according to the sex and familiarity of a donor, preferring samples from familiar animals of the opposite sex (caught within $25 \mathrm{~m}$ of the subject). They showed no bias in manipulating samples from different conspecifics. Given the frequency with which moist fresh faeces stuck to mice as they passed, the results suggest that manipulation is a hygienic response to move sticky faeces away from pathways and resting sites. However, faeces are likely to play an important role in providing social information in $M$. spretus populations.
\end{abstract}

Mus spretus Lataste is an aboriginal species of house mouse, closely related to the much more familiar pest of human habitation $M$. domesticus, but living in relatively undisturbed grassland and woodland around the western Mediterranean (Marshall \& Sage 1981). During a study of the social responses of wild $M$. spretus to conspecifics and their tunnels (Hurst et al. 1994), we observed an unusual pattern of response when they encountered previously deposited faeces on the ground. While confined temporarily in test arenas (individually or in dyads), both males and females frequently picked up their faeces in their mouths and moved them several centimetres, or rolled them forwards or sideways with the tip of the snout, sometimes repeatedly. Most movements were less then $10 \mathrm{~cm}$, although they often involved the repeated re-siting of a pellet within a small area before.it was left alone and subsequently ignored. Mice sitting in corners often moved their faeces a few centimetres in front of themselves so that they sat surrounded by one or more pellets. When dyads of mice were together in the test arena, however, it was not clear whether they moved only their own faecal pellets or also those of the other mouse.

Many species of rodents deposit their faeces in specific sites or latrines, probably as signals for social communication (reviewed by Brown 1985). Faecal manipulation with both the mouth and forepaws has been observed in bank voles, Clethrionomys glareolus (Rosenfeld \& Rasmont 1991) and hamsters, Mesocricetus auratus (Johnston et al. 1993), while hamsters keep their nests clean by tossing their faeces out by mouth (S. Gray, personal communication). However, there appear to be no previous records of rodents deliberately moving deposited faeces around while exploring their environment as $M$. spretus do. Their behaviour could have a number of potential functions. The re-siting of odorous faeces may be important for crypsis, hiding their activity from predators or conspecifics, or play a role in social communication (e.g. providing territorial or location markers). Alternatively, this could be a hygienic response, especially since $M$. spretus are known to carry a number of helminth parasites which are transmitted through faecal eggs (Behnke et al. 
1993). To investigate these potential functions further, a pilot experiment was conducted followed by a more extensive experiment a year later.

\section{EXPERIMENT 1}

To examine whether mice (1) move the faeces of conspecifics as well as their own, (2) appear to discriminate between faeces from different conspecifics by differential manipulation or investigation of their odours, and (3) respond differently according to the physical location of a sample (e.g. in corners or along a pathway), the behaviour of four wild-caught $M$. spretus was measured towards their own and each other's faeces.

\section{Methods}

The subjects were two male-female pairs of $M$. spretus caught in two well-separated grassland areas approximately $3 \mathrm{~km}$ apart near Lisbon, Portugal. Pairs were potential mates that had been caught in the same tunnel system and had shown affiliative behaviour (sitting together and allogrooming) with no sign of aggression or defence during a 3-min trial of their social response (Hurst et al. 1994). In contrast, all four mice showed cautious and defensive responses towards the other two unfamiliar subjects regardless of their sex, with aggression arising rapidly between the two males.

After capture, the mice were housed individually in clean polypropylene cages $(30 \times 13 \times$ $12 \mathrm{~cm}$ ) containing sawdust and dried grass bedding. Laboratory mouse food pellets (Banton \& Kingman, U.K.), wheat grain and water were provided ad libitum. Cages were housed under dim red light in a darkened room where tests were carried out over 5 days. Each day, the mice were housed on clean sawdust and the faeces deposited in their home cage were collected for testing. Trials were carried out during the early evening (1800-2200 hours) in a varnished plywood arena $(60 \times 60 \times 60 \mathrm{~cm})$, cleaned between trials with detergent then alcohol.

Each mouse was presented with faeces from all four subjects simultaneously. Prior to mtroduction, single faecal pellets (less than $12 \mathrm{~h}$ old) from each of the subjects were placed in scparatc corners, along the side walls and in the centre of the arena, as shown in Fig. 1. Samples from

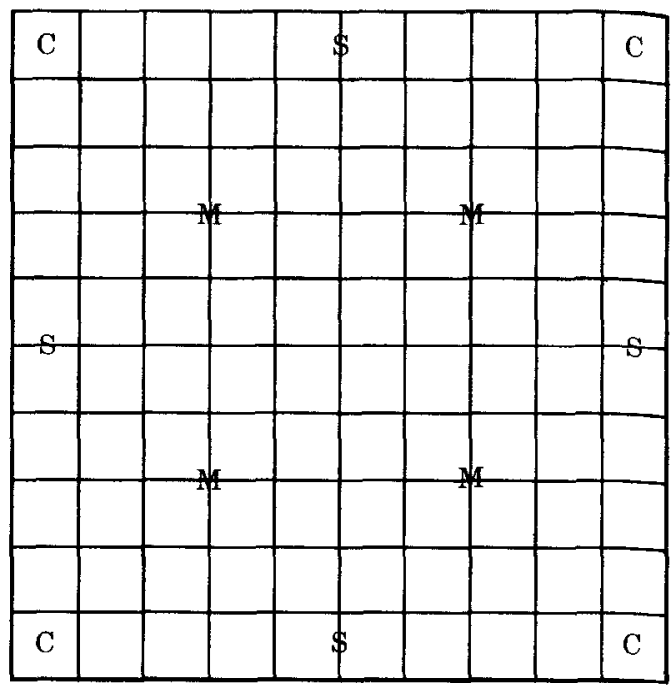

Figure 1. Layout of faecal pellets in experiment 1. A $10 \times 10$ grid of $6-\mathrm{cm}$ squares was drawn on the floor of the enclosure to aid the recording of faecal locations and movements. One of each of the four sample types was placed in a separate corner $(C), 3 \mathrm{~cm}$ from a side wall $(\mathrm{S})$ and in the middle $(\mathrm{M})$ of the arena immediately prior to each trial.

different individuals were handled with clean forceps to avoid contamination and their positions within each of the three locations randomized in each trial to avoid any site bias. Subjects were introduced into the centre of the arena from a clean Perspex tube to avoid handling stress (see Hurst et al. 1994) and their behaviour recorded for $5 \mathrm{~min}$. An observer and a recorder scored (1) every encounter with each faecal sample, counted when the tip of a mouse's nose passed over the sample, (2) investigation when the mouse paused with nose over the sample, usually making sniffing movements, (3) manipulation when the moușe rolled the sample with its snout or picked it up by mouth, together with the distance moved in centimetres, and (4) if a mouse sat within $3 \mathrm{~cm}$ of a sample for more than $10 \mathrm{~s}$. These categories of behaviour were also scored with respect to any fresh faeces deposited by the subject during a trial together with their location. Five trials were carried out on successive days for each subject. Data are presented as means \pm SE per manipulation or trial, or as a proportion of encounters with faecal samples totalled across all trials. At the end of the experiment mice were released at their site of capture. 
Table I. Response to introduced faeces, experiment 1

\begin{tabular}{|c|c|c|c|c|}
\hline & \multicolumn{2}{|c|}{ Familiar } & \multicolumn{2}{|c|}{ Unfamiliar } \\
\hline & Own* & Mate & Same sex & Opposite sex \\
\hline Encounters & 165 & 149 & 171 & 165 \\
\hline Proportion manipulated $(\%)$ & $6 \cdot 1$ & $3 \cdot 4$ & $3 \cdot 5$ & $4 \cdot 8$ \\
\hline Distance moved $(\mathrm{cm})$ & $3 \cdot 9 \pm 1 \cdot 0$ & $5 \cdot 6 \pm 1 \cdot 8$ & $2 \cdot 3 \pm 0 \cdot 8$ & $5 \cdot 1 \pm 1 \cdot 5$ \\
\hline Investigation only $(\%)$ & $24-8$ & $41 \cdot 6$ & $31 \cdot 1$ & $31 \cdot 4$ \\
\hline
\end{tabular}

*Not including fresh faeces deposited during the trial.

\section{Results}

All four subjects showed very similar behaviour, summarized in Table I, with no change in their response apparent in successive trials. When manipulating faeces, the mice did not appear to discriminate between samples from the four different types of donor, although we did not have sufficient data for statistical analysis. Faeces were moved in 29 of 650 encounters $(4.5 \%)$ over all trials, with very similar rates between subjects $(5 \cdot 8 \%, 3 \cdot 1 \%, 6.5 \%, 2.0 \%)$. All manipulations involved some re-siting of the sample, although only over short distances $(4 \cdot 2 \pm 0 \cdot 7 \mathrm{~cm}$ per move). Location appeared to be an important factor since faecal samples in the corners of the arena were more likely to be moved (in $7.9 \%$ of encounters) than those along the side walls $(1.7 \%)$ or in the middle $(2.4 \%)$ of the arena. Subjects were much more likely to sit in the corners and thus by these faeces than elsewhere (in 18\% of encounters versus $1 \%$ of side wall and none by central faeces), and so had much greater contact with the manipulated corner faeces.

The main difference in manipulation was between experimental samples and fresh faeces deposited during the trial $(1 \cdot 6 \pm 0.3$ faeces deposited per trial). While samples collected from the subject's own holding cage were moved in only $6.1 \%$ of encounters, freshly deposited faeces were moved in $52.9 \%$ of encounters, often with repeated movement of the same faecal deposit. Generally, these were not manipulated immediately after deposition but when encountered as the mouse.moved around the enclosure. The short distances involved $(4.9 \pm 0.7 \mathrm{~cm})$ were similar to those of experimental samples. Almost all fresh faeces were deposited near to the experimental samples, with $77.3 \%$ deposited in corners and a further $13.6 \%$ within $3 \mathrm{~cm}$ of experimental faeces sited along the side walls. However, this pattern may simply reflect the fact that the mice spent most of their time in corners or moving around the side walls. Faecal deposition did not appear to be biased towards samples from different donors.

Faeces were investigated much more frequently than they were moved (Table I). The mice were most likely to investigate samples from a familiar mate and least likely to investigate their own, with an intermediate response towards those from unfamiliar mice of either sex (Table I). This was due mostly to discrimination between faeces encountered in corners, where mice were more likely to pause. Considering responses of the two sexes separately, only the males appeared to discriminate between faeces in all locations, with a strong preference for samples from a presumed mate (investigating in $42 \%$ of encounters versus $24.5 \%$ for other samples). Females appeared to discriminate between corner samples only, owing to more frequent investigation of those from males (familiar and unfamiliar, 53\%) than from females $(29 \%)$. In both sexes, own freshly deposited faeces stimulated infrequent investigation ( $21 \%$ of encounters) similar to samples collected from the subject's holding cage $(25 \%$ of encounters).

\section{EXPERIMENT 2}

It was not clear from the results of experiment 1 whether the much more frequent manipulation of a subject's own freshly deposited faeces was stimulated simply by the freshness of the deposit, or also because they originated from the subject itself. In addition, the act of deposition might have played a part in their response. Faeces deposited during trials were larger and more moist than those collected from their cages which, 
although less than $12 \mathrm{~h}$ old, had dried in the open air. The fresh faeces were much more likely to become squashed and to stick to the feet and fur as a subject passed. Samples collected from home cages could also have been contaminated by odours from the sawdust cage substrate. The following year, we conducted a second experiment to examine faecal manipulation in more detail using fresher, uncontaminated faeces and a larger number of subjects and sample donors. In addition, we examined whether the eggs of parasitic tapeworms (Taenia taeniaeformis and several Hymenolepis spp.; Behnke et al. 1993) present in the faeces and likely to be transmitted by oral manipulation influenced the response.

\section{Methods}

Individual $M$. spretus were confronted with a single faecal bolus in a clean Perspex tunnel ( $38 \mathrm{~cm}$ long, $3 \mathrm{~cm}$ diameter) fitted with mesh caps at either end. Subjects were 14 adult males and 11 adult females caught from the main Portuguese study site described in Hurst et al. (1994) and had been housed singly in captivity as in experiment 1 , for 1-8 days prior to testing. The response of both males and females was tested towards their own faeces $(N=14,11)$, those from a conspecific of the same sex $(N=14,17)$, or from a conspecific of the opposite sex $(N=17,13)$. All subjects had encountered the conspecific donor previously within a similar tunnel during a brief 5-min trial, and had been held in an empty tunnel previously used by the donor for a further $2 \times 2 \mathrm{~min}$, during another (unpublished) experiment. Their immediate response on encountering each conspecific donor was thus known and will be summarized here as aggressive, defensive or ambivalent (see Hurst et al. 1994 for further details on classification of social response). No dyad was used more than once, with individual subjects used in $3.6 \pm 0.4$ trials and individual donors in $3.4 \pm 0.5$ trials to avoid habituation or bias caused by the behaviour or faecal samples of a few individuals. Subjects were released at their site of capture at the end of the experiment except for a sample of nine individuals which were killed to identify their internal parasites (Behnke et al. 1993).

\section{Test procedure}

Faccal samples wcrc collected by eonfining donors for $5 \mathrm{~min}$ in a clean tunnel $(19 \mathrm{~cm}$ long) which they entered readily and any faeces produced were then transferred immediately to a sealed glass vial and used within $3 \mathrm{~h}$. All samples retained the same moist stickiness and size of faeces just deposited. Tests were conducted in a dark room with a dim red light centred over a clean test tunnel, positioned at observer eye-level so that behaviour could be recorded in detail. A single faecal pellet was placed $8 \mathrm{~cm}$ from one end of the tunncl and the subject introduced into the opposite end (from another clean tunnel to avoid handling stress) for a 5-min trial. Two observers measured the frequency, duration and type of any manipulation involving the nose or mouth, the distance and direction the sample was moved, the frequency and duration of any investigation, and the frequency of encounters when the sample was passed without manipulation or investigation. Accidental movement as a mouse passed the sample was not recorded. The test tunnel was mounted on a sheet of lined paper and distances measured as the number of $8-\mathrm{mm}$ lines moved. We also recorded the number of faeces deposited by the subject within $8 \mathrm{~mm}$ of the sample or elsewhere in the tunnel, and any manipulations of these faeces. The rate of faeces deposition was also measured in control trials ( 10 male, 10 female) in an empty tunnel to check whether the presence of other faeces influenced further deposition. Trials were conducted in random order between 1300 and 2000 hours over an 8-day period, with at least 60 min between trials if a subject was used more than once on the same day (13\% of all trials).

Subjects and donors were caught at varying distances from each other $(0-140 \mathrm{~m})$ and thus were likely to differ in prior familiarity with each other's odours. For analysis, dyads caught within $25 \mathrm{~m}$ of each other were classified arbitrarily as from the same area. Traps within this distance were always located in the same set of grass tunnels centred around a bank or clump of bushes, while traps separated by habitat containing no signs of mice (spare grass or a cliff) were always more than $35 \mathrm{~m}$ apart. To assess whether mice responded differently to samples containing parasites likely to be transmitted by oral manipulation, or whether there was any difference in behaviour between infected and non-infected subjects, additional faecal samples from each individual were examined for the presence and number of tapeworm cggs (Behnke et al. 1993). Mice were classified as infected ( $29 \%$ of males, $45 \%$ of 
Table II. Response to introduced faeces, experiment $2(\bar{X} \pm$ SE per trial)

\begin{tabular}{|c|c|c|c|c|}
\hline \multirow{2}{*}{\multicolumn{2}{|c|}{ Own }} & \multicolumn{2}{|c|}{ Conspecific } & \multirow{2}{*}{$\begin{array}{l}\text { Own versus } \\
\text { conspecifict }\end{array}$} \\
\hline & & Same sex & Opposite sex & \\
\hline \multicolumn{5}{|c|}{ Manipulation (" $/ \%$ encounters) } \\
\hline Males & $24 \cdot 0 \pm 7 \cdot 2$ & $9 \cdot 1 \pm 4 \cdot 2$ & $4 \cdot 5 \pm 2 \cdot 1$ & $* * *$ \\
\hline Females & $15 \cdot 0 \pm \quad 4.9$ & $8 \cdot 1 \pm 3 \cdot 7$ & $10 \cdot 0 \pm 5.4$ & \\
\hline \multicolumn{5}{|c|}{ Manipulation duration (s) } \\
\hline Males & $2 \cdot 4 \pm 0 \cdot 8$ & $0 \cdot 6 \pm 0 \cdot 3$ & $0.4 \pm 0.2$ & $* *$ \\
\hline Females & $0.7 \pm 0.3$ & $0.6 \pm 0.3$ & $0.5 \pm 0.3$ & \\
\hline \multicolumn{5}{|c|}{ Distance moved (mm) } \\
\hline Males & $6 \cdot 4 \pm 2 \cdot 1$ & $1 \cdot 7 \pm 1 \cdot 0$ & $0.8 \pm 0.6$ & $* * *$ \\
\hline Females & $2 \cdot 0 \pm 0.7$ & $1.4 \pm 0.7$ & $1 \cdot 1 \pm 0.8$ & \\
\hline \multicolumn{5}{|l|}{ Faeces deposited $\ddagger$} \\
\hline Males & $2 \cdot 3 \pm 0 \cdot 5$ & $1.8 \pm 0.4$ & $3.1 \pm 0.5$ & Ns \\
\hline Females & $2.5 \pm 0.7$ & $1 \cdot 9 \pm 0 \cdot 4$ & $1 \cdot 9 \pm 1 \cdot 3$ & \\
\hline \multicolumn{5}{|c|}{ Investigation ( $\%$ encounters) } \\
\hline Males & $50 \cdot 1 \pm 6 \cdot 6$ & $79.9 \pm 4.8$ & $86.7 \pm 3.4$ & $* * *$ \\
\hline Females & $47 \cdot 2 \pm 10 \cdot 4$ & $75.4 \pm 7.7$ & $64 \cdot 0 \pm 11 \cdot 1$ & \\
\hline \multicolumn{5}{|c|}{ Investigation duration (s) } \\
\hline Males & $2.4 \pm 0.4$ & $3.6 \pm 0.9$ & $3.7 \pm 0.5$ & vis \\
\hline Females & $1.9 \pm 0.4$ & $2 \cdot 4 \pm 0 \cdot 6$ & $2 \cdot 2 \pm 0.6$ & \\
\hline \multicolumn{5}{|c|}{ Passing (\% encounters) } \\
\hline Males & $25 \cdot 9 \pm 8 \cdot 2$ & $11 \cdot 0 \pm 4 \cdot 1$ & $8.8 \pm 3.6$ & * \\
\hline Females & $37 \cdot 7 \pm 11 \cdot 1$ & $16.5=7 \cdot 0$ & $26.0 \pm 10.9$ & \\
\hline \multicolumn{5}{|c|}{ Investigation (s per encounter) } \\
\hline Same area & - & $0.45 上 0.08$ & $0.75 \pm 0.19$ & - \\
\hline Different area & - & $0 \cdot 40 \pm 0 \cdot 03$ & $0.50 \pm 0.09$ & \\
\hline
\end{tabular}

$\mp$ Wilcoxon matched-set tests (both sexes combined): $* P<0 \cdot 05 ; * * P<0 \cdot 01 ; * * * P<0 \cdot 001$. Mann-Whitney tests of the mean response per individual confirmed that there were no significant differences in response between males and females.

\#Similar numbers were deposited in control trials when no test sample was present (males $3 \cdot 0 \pm 0 \cdot 7$, females $1 \cdot 6 \pm 0 \cdot 5$ ).

females) or non-infected; samples from infected mice contained 1400-73000 eggs/g.

\section{Data analysis}

Frequencies were converted to rates per $5 \mathrm{~min}$ to take into account trials that were curtailed when the test sample became confused with freshly deposited faeces (7/86 trials) or was pushed out of the tunnel $(2 / 86)$. Wilcoxon two-sample matched-set tests (Meddis 1984) examined whether mice (1) manipulated their own faeces more than those of a conspecific (all types), and (2) investigated a conspecific's faeces more than their own, after Mann-Whitney $U$-tests confirmed that there were no significant differences in the mean response to own-mean response to conspecific's faeces between individual males and females. Discrimination in responsc to facces from different conspecifics according to their sex and capture site were also tested using Mann-Whitney tests, while Kruskal-Wallis non-parametric analysis of variance assessed discrimination according to a subject's social response to the faecal donor.

\section{Results}

Response to own versus conspecific faeces

- Subjects were more likely to manipulate their own faeces than others even when all were fresh. although there appeared to be no qualitative difference in their manipulatory behaviour. The test sample was manipulated in $56^{\%} \%$ of trials when this was the subject's own but in only $26.2 \%$ when it came from a conspecific $\left(\chi^{2}=6.9, d f=1\right.$. $P<0.01$ ), and their own sample was manipulated in a greater proportion of encounters per trial (Table II). Discrimination was strongcst anmong males $(z=2 \cdot 77, P<0.01)$ although females also 
showed a significant bias $(z=1.71, P<0.05)$. Mice of both sexes thus spent more time manipulating their own sample and moved it further during a trial (Table II). The mean distance moved per manipulation also was greater $(z=2.61, P<0.01)$ but no difference was detected in the mean duration of each manipulation $(z=0 \cdot 027$, NS). In contrast to experiment 1 , the sample was re-sited within the tunnel in most but not all cases (in 77 of 84 instances when samples were manipulated), on average by $2.6 \mathrm{~cm}$ per move. Regardless of their origin, samples were picked up in the mouth and carried $(74 \%$ of manipulations) much more frequently than rolled $(18 \% ; z=3.69, P<0.001)$. In all but two cases, the sample was moved forwards rather than backwards along the tunnel. The mice were never observed to lick faeces or manipulate them with their forepaws.

Test samples collected from the subject itself were manipulated at a similar rate (males $2 \cdot 5 \pm 1 \cdot 1$, females $1.2 \pm 0.4$ moves per trial) as new faeces deposited during the trial (males $1 \cdot 7 \pm 0 \cdot 4$, females $1.5 \pm 0.4$ moves per new faeces per trial, but note movement rates were underestimated since new faeces were not present for the whole trial). Subjects did not appear to deposit new faeces deliberately near the test sample. However, in 15 of 86 trials we noted that deposited faeces were carried or rolled to the sample or to other new faeces, often creating a small pile.

In contrast to manipulation, a subject's own faeces stimulated much less frequent investigation than those of a conspecific, a difference that was particularly pronounced among males (Table II). This was not simply a reflection of the difference in manipulatory behaviour as both males and females were more likely to pass their own faeces without investigation or manipulation than those of a conspecific (Table II). However, there was no significant difference in the duration of investigation (per investigation or per trial).

\section{Effect of tapeworm infection}

There were no significant differences in response to infected versus non-infected faeces from conspecifics, and the infection status of subjects did not affect their willingness to manipulate faeces. However, infected mice of both sexes investigated their own (infected) faeces for longer per trial $(3.0 \pm 0.3 \mathrm{~s}, N=10)$ than equivalent investigation by non-infected mice $(1 \cdot 6 \pm 0 \cdot 3 \mathrm{~s}, N=15 ; z=2 \cdot 33$,
$P<0.05$ ), suggesting that they detected a difference in their own familiar odour.

\section{Discrimination between conspecific faeces}

Manipulation of conspecific faeces did not vary significantly according to the sex of the subject or odour donor, their social response when they met the donor (classified as aggressive, defensive or ambivalent), or the closeness of their capture sites. However, differences in investigation again suggested that mice could discriminate between faeces according to the sex and familiarity of a donor. Consistent with experiment 1 , there was more investigation per encounter when samples came from conspecifics caught from the same area and were potentially familiar to the subject than from those caught at a distance $(z=2 \cdot 29, P<0.05)$. This was due to greater investigation per encounter with faeces from familiar animals of the opposite sex (Table II).

\section{DISCUSSION}

The manipulatory behaviour shown by $M$. spretus was clearly designed to re-site their faeces, either by carrying or rolling them and occasionally even throwing them forwards. There was no sign of any licking, mouthing or holding faeces in the forepaws which appears to be a type of social marking behaviour shown by bank voles (Rozenfeld \& Rasmont 1991). Furthermore, bank voles handle only the faeces of other conspecifics and not their own (Rozenfeld \& Rasmont 1991). The simplest explanation for the function of faecal manipulation in $M$. spretus is that it is a hygienic response to remove sticky fresh faeces away from pathways and resting sites. The frequency with which fresh deposits stuck to the fur and feet as mice brushed past in our tests would certainly justify such a response. Their faeces appeared to be more moist than those of $M$. domesticus living commensally with humans (personal observation), probably reflecting a higher proportion of living plant and insect material in their diet rather than dry stored products. This may explain why $M$. spretus show a behaviour pattern that we have never observed among $M$. domesticus, despite many years of research into their behaviour.

Within their natural surroundings in the grassy undergrowth, a pellet rolled forward by a few 
centimetres is likely to be pushed through the grass stems out of contact, while those in more solid tunnels may be carried forwards until they too drop out of the way. Mice generally showed no further interest in faeces once they had been taken to one end of our test tunnel and, on several occasions, even pushed them through the end mesh. If they were attempting to remove evidence of their recent location from predators (or conspecifics), they should have removed any signs of recent activity in the area, regardless of the depositor, and moved the tell-tale odours much further. The finding that mice showed a strong, although not exclusive, preference for moving their own deposits is not surprising if their usual habit is to move them out of the way soon after dropping them. This is likely to lead to rapid recognition and a conditioned response to the odour of their own fresh deposits. The faeces of other mice, however, might also be moved if moist and in the way. It seems unlikely that their discrimination was an attempt to avoid oral contact with potentially infective material from other hosts. Their discrimination would need to be much stronger to avoid such risk and, even though they appeared to detect a different odour when their own faeces were infected, we found no evidence that they were influenced by the presence of tapeworm eggs in faeces.

Differences in their investigation of faeces according to the relative capture site and sex of subjects and donors suggested that $M$. spretus could discriminate between faecal odours from different individuals. Commensal house mice do not show the same interest in faecal deposits and fail to discriminate between those from different individuals or classes, using mainly urinary odours for communication (see Brown 1985 for a review). In contrast, the woodmouse, Apodemus sylvaticus, can be trained to discriminate between individual conspecifics using faecal odours (Wolton 1984), while bank voles behave differently towards faeces from bank voles or other species (Microtus arvalis or woodmice), and towards their own versus those of other conspecifics (Rozenfeld \& Rasmont 1991). Faeces may provide more persistent odour markers under open field conditions than urine marks (Wolton 1985) which are deposited on comparatively stable and covered surfaces by domestic house mice (Hurst 1987). Faeces are thus likely to play a role in providing social information in $M$. spretus populations, but it seems unlikely that the main function of the manipulatory behaviour is to place social signals in specific sites. Mus spretus rarely manipulated drier samples even though patterns of investigation suggested that these still emanated their donor's odours, and faeces from conspecifics were frequently moved in a similar way to their own when fresh. This does not rule out the possibility that faecal signals were re-sited to advertise the presence of $M$. spretus to other species. In our study sites, $M$. spretus shared their tunnel systems and pathways with woodmice and with shrews (Crocidura russula). Frequently dropping and rolling fresh wet faeces on the substrate has the potential to smear their odour over a wider area, although the significance of this remains to be tested.

\section{ACKNOWLEDGMENTS}

We thank Armin Pircher, Warden of the Quinta de Sao Pedro Research Station, for his support and hospitality; Mandy Kingston, Rebecca Luck, Karin Sorensen, Lee Hayden, Charlotte Nevison, Pippa Jones, Richard Holland and Robin Dryden for helping to catch the mice and collect samples; and Dr J. Behnke, John Owen, Jennifer Collinson and Caroline Crean for the tapeworm egg counts. J.L.H. was supported by an Advanced Research Fellowship and J.S. by a postgraduate studentship from the Science and Engineering Research Council. The work was carried out during two Terrestrial Zoology field courses funded by the University of Nottingham.

\section{REFERENCES}

Behnke, J. M., Barnard, C., Hurst, J. L., McGregor, P. K., Gilbert, F. \& Lewis, J. W. 1993. The prevalence and intensity of infection with helminth parasites in Mas spretus from the Setubal Peninsula of Portugal. J. Helminthol., 67, 115-122.

Brown, R. E. 1985. The rodent II: suborder Myomorpha. In: Social Odours in Mammals, Vol. 1 (Ed. by R. E. Brown \& D. W. Macdonald), pp. 345-457. Oxford: Clarendon Press.

Hurst, J. L. 1987. The functions of urine marking in a free-living population of house mice, Mus domesticus Rutty. Anim. Behav, 35, 1433-1442.

Hurst, J. L., Hayden, L., Kingston. M., Luck, R. \& Sorensen, K. 1994. Response of the aboriginal house mouse Mus spretus Lataste to tunnels bearing the odours of conspecifics. Anim. Behav., 48, 1219-1229. 
Juhuston, R. E., Derzie, A., Chiang, G., Jernigan, P. \& Ho-Chang, L. 1993. Individual scent signatures in golden hamsters: evidence for specialization of function. Anim. Behav, 45, 1061-1070.

Marshall, J. T. \& Sage, R. D. 1981. Taxonomy of the house mouse. Symp. zool. Soc. Lond., 47, 15-25.

Meddis, R. 1984. Statistics Using Ranks, Oxford: Blackwell Scientific Publications.
Rozenfeld, F. M. \& Rasmont, R. 1991. Odour cue recognition by dominant male bank voles, Clethrionomys glareolus. Anim. Behav., 41, 839-850.

Wolton, R. J. 1984. Individual recognition by olfaction in the wood mouse, Apodemus sylvaticus. Behaviour, 88, 191-199.

Wolton, R. J. 1985. A possible role for faeces in rangemarking by the wood mouse, Apodemus sylvaticus. J. Zool. Lond., 206, 286-291. 